\title{
The diffusion of grassroots innovations for sustainability in Italy and Great Britain: an exploratory spatial data analysis
}

\author{
GIUSEPPE FEOLA AND ANISA BUTT \\ Department of Geography and Environmental Science, University of Reading, Whiteknights, \\ Reading RG6 6AB \\ E-mail: g.feola@reading.ac.uk
}

This paper was accepted for publication in June 2015

\begin{abstract}
Little research so far has been devoted to understanding the diffusion of grassroots innovation for sustainability across space. This paper explores and compares the spatial diffusion of two networks of grassroots innovations, the Transition Towns Network (TTN) and Gruppi di Acquisto Solidale (Solidarity Purchasing Groups - GAS), in Great Britain and Italy. Spatio-temporal diffusion data were mined from available datasets, and patterns of diffusion were uncovered through an exploratory data analysis. The analysis shows that GAS and TTN diffusion in Italy and Great Britain is spatially structured, and that the spatial structure has changed over time. TTN has diffused differently in Great Britain and Italy, while GAS and TTN have diffused similarly in central Italy. The uneven diffusion of these grassroots networks on the one hand challenges current narratives on the momentum of grassroots innovations, but on the other highlights important issues in the geography of grassroots innovations for sustainability, such as cross-movement transfers and collaborations, institutional thickness, and interplay of different proximities in grassroots innovation diffusion.
\end{abstract}

KEY WORDS: grassroots innovations, sustainability, Transition Network, Solidarity Purchasing Groups, alternative economies, diffusion

\section{Introduction}

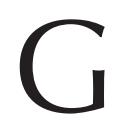

rassroots movements can be influential agents of change in a transition to sustainability. While a long tradition of social movement studies has investigated the role of community action in relation to environmental change and sustainable development, various scholars have more recently highlighted the specific contribution of grassroots movements to innovation towards sustainability (Seyfang 2009; Peters et al. 2010). This theoretical development has combined social movement studies and Transition Theory, thus helping to conceptualise grassroots movements as laboratories of innovative practices and alternative social, cultural and economic models that can prefigure and help activate pathways to sustainable futures (Seyfang and Smith 2007). It is now recognised that grassroots movements, enlaced in complex relations with, and at times in opposition to, 'conventional' governmental and corporate actors, can have a transformative power, and that their role is in fact fundamental for a just transition to sustainability (Ornetzeder and Rohracher 2013; Smith and Seyfang 2013, Leach et al. 2012).

There is evidence that the geography of grassroots movements matters (Nicholls 2007), and issues of place, space and scale have been central in the discussion of the role of grassroots movements in the emergence of and transition to alternative economies (Bailey et al. 2010). For example, North (2010a) and Wilson (2012) questioned localisation as a strategy to achieve resilience of local communities in the face of global environmental change, and explored the multilevel interactions and interdependencies of local initiatives and the global economic system. Pickerill and Maxey (2009), among others, discussed the importance of scale and sense of place in collective community action and grassroots innovation. With reference to the Transition Movement, Feola and Nunes (2014) suggested that direct interaction between local transition initiatives, which is facilitated by their geographical proximity, is an important factor of success. The conditions for success and failure of the Transition Movement have also been discussed by North and Longhurst (2013), who suggested that 
urban, as opposed to rural, places may offer more conducive conditions to local transition initiatives.

However, while it is apparent that the geography of grassroots innovations for sustainability is important in explaining their emergence and development, little research has been focussed particularly on their spatial diffusion. Social movement diffusion studies have mostly investigated the social mechanisms rather than the spatial structure of diffusion (e.g. Strang and Soule 1998; Shawki 2013; Walsh-Russo 2014). Furthermore, comparative studies of sustainability transitions are lacking (Strang and Soule 1998), and as argued by Coenen et al. (2012), this may be one of the reasons why the geographical dimension of sustainability transition has generally been overlooked.

Indeed, a growing body of literature has recently applied Transition Theory to conceptualise and understand the dynamics of socio-technical change generated by grassroots movements that promote bottom-up innovation for sustainability (Seyfang and Smith 2007). This theoretical perspective bridges the innovation and community action strands of academic theory, thus providing a nuanced and multilayered theoretical approach to grassroots innovations towards sustainability.

Transition Theory has traditionally had blindspots on the geographical dimension of transition. Only recently have some scholars stressed the importance of understanding sustainability transition as a geographical process, and have started to introduce a new vocabulary in Transition Theory that encompasses such concepts as location, landscape, territoriality, uneven development, scaling, and embeddedness (Coenen et al. 2012; Markard et al. 2012; Raven et al. 2012; Truffer and Coenen 2012; Bridge et al. 2013; Caprotti and Bailey 2014). While this cross-fertilisation between Transition Theory and geographical traditions such as economic geography has generated some promising developments, research has focused on particular socio-technical systems (e.g. Newton and Newman 2013), or the so-called green economy (e.g. Gibbs and $\mathrm{O}^{\prime} \mathrm{Neill}$ 2014), and has largely overlooked grassroots innovations and alternative economies (Schulz and Bailey 2014). As Caprotti and Bailey (2014) observe, such a narrow focus is constraining, in that it reduces the space for alternative ways of envisioning the future and tends to align itself with 'key tenets and mainstream discursive strands which construct the green economy as a socio-economic and techno-environmental project that is inevitably and unquestioningly based on the same concepts of growth, production, and consumerism that characterized the old economies of neoliberal capitalism' (2014, 5; see also Schulz and Bailey 2014).

Against this backdrop, this paper explores and compares the spatial diffusion of two networks of grassroots innovations in Great Britain and Italy. It aims to uncover patterns of grassroots innovation diffusion in space and over time, and thus to advance our understanding of the geographical dimension of grassroots-driven sustainability transitions. By providing a descriptive analysis of spatial patterns of grassroots innovation diffusion, this paper contributes to the literature on social movements, grassroots innovations for sustainability, and sustainability transitions, and can inform future geographical analysis of the emergence and diffusion of alternatives to unsustainable economies of neoliberal capitalism.

\section{Academic context}

Following McAdam et al. (2001, 68), social movement diffusion can be defined as the 'transfer in the same or similar shape of forms and claims of contention across space or across sectors and ideological divides'. Perhaps surprisingly, only a few studies have focused on the movement 'across space' $^{\prime}$ of the diffusion of grassroots innovations for sustainability, and those who did have done so more theoretically than empirically. For instance, Bailey et al. (2010) and Scott-Cato and Hillier (2010) proposed that social innovation towards sustainability spreads in a rhizome-like fashion, which emphasises networking, relationality and transversality rather than spatial proximity, and random connectivity rather than patterns and structure in the process of diffusion. Bailey et al. also proposed that the rhizome-like diffusion of the Transition Network may be linked to three geographically determined factors, namely the societal acceptance of environmental threats, the influence of 'gatekeepers' (e.g. local media and politicians) and local leaders. Similarly, Rob Hopkins, the co-founder of the Transition Movement, used the metaphor of viral infection in a famous speech to describe the rapid diffusion of the Transition Network (Hopkins 2009). However, no study so far has empirically investigated the spatial pattern of diffusion of this network.

Indeed, the literature has shown that processes of social movement diffusion are often spatially structured. In a study of trade unions in Sweden, Hedström (1994) found that because the movement diffused through social networks and these are shaped by the actors' (i.e. nodes') spatial locations, the process of diffusion was spatially structured. Andrews and Biggs (2006), on the other hand, found that the diffusion of protest in the 1960s in the USA occurred through mass media and local contextual factors, such as a large student population and more favourable political opportunities (e.g. the configuration of institutions). In this case, the diffusion was spatially structured, but the spatial structure was determined by the uneven distribution of those contextual factors rather than the spatial location of actors of relevant social networks. 
Along these lines, Nicholls $(2007,607)$ stressed that social movements are 'horizontal and loosely constituted networks' that 'generate organizational and relational dynamics that are different from those found in hierarchical and centralized parties and organizations'. Nicholls maintained, nevertheless, that successful social movements tend to depend on a combination of territorially intensive relations to secure resources (e.g. reputation, tacit knowledge) (see also Ornetzeder and Rohracher 2013), and geographically extensive relations that enable the circulation of other resources (e.g. information). Feola and Nunes (2014) suggested that something similar may be occurring in transnational networks of grassroots innovations like the Transition Movement, in which local transition initiatives remain determined by situated processes, but benefit from the interaction and support of other initiatives and the ability of national and transnational network hubs to generalise and socialise organisational principles derived from 'unique' local experiences elsewhere.

Tarrow (2005) has further addressed the diffusion of transnational movements across countries. Tarrow identified three diffusion pathways, namely relational (i.e. via interpersonal contact and communication), non-relational (i.e. via the media) and mediated (i.e. via movement brokers) diffusion, all of which entail the 'emulation of local forms of collective action in other places' (Tarrow 2005, 102; see also Tarrow 2010). Building on Tarrow's work, Shawki (2013) showed that in grassroots innovations for sustainability such as the Transition and Solidarity Economy Movements, a combination of the three diffusion pathways operate. While non-relational diffusion makes activists initially aware of initiatives in other countries, relational diffusion makes possible the in-depth exchange of ideas, information, and experiences (Shawki 2013). Shawki particularly stressed the role of movement brokers and translators in mediated diffusion, whereby translators can be individuals or organisations that connect local and global activist communities. This is often done by transnational network hubs through the internet, social events and training (Feola 2014; Feola and Nunes 2014).

Thus, while 'one of the most common findings in diffusion research is that spatially proximate actors influence each other' (Strang and Soule 1998:275), the literature on the transnational diffusion of grassroots innovations challenges spatial proximity as a key enabler of diffusion.

Strang and Soule (1998), for example, argued that spatial proximity may facilitate various types of interaction and influence that lead to social movement diffusion, but that other mechanisms may be at play and involve social media, change agents, strong and weak social ties (related to cohesion and information respectively), and prestige (see also Andrews and Biggs 2006; Feola and Nunes 2014).
Boschma (2005) distinguished five types of proximity that may be involved in innovation processes, namely cognitive, social, institutional, organisational and geographical (see also Raven et al. 2012). They correspond to five different processes, namely learning, decoupling, institutionalisation, integration, and agglomeration respectively (Balland et al. 2014). This suggests that geographical, or spatial, proximity may not be a necessary and sufficient condition for successful grassroots innovation diffusion. Furthermore, Boschma's contribution is in line with work on social movement diffusion, in which cultural proximity, and the active and constitutive process of creating that type of proximity, is thought to play a fundamental role in social movement diffusion. Such a process, often termed 'framing' or 'theorisation', entails the social construction and attribution of similarity when social networks and social ties are absent (Soule 2004; Shawki 2013), and is key in both relational (i.e. through personal contact) and nonrelational (i.e. through channels of information diffusion, online media) forms of connection between seekers and transmitters of innovation. Transnational movements create collective identities that support transnational as well as local actions. Importantly, transnational diffusion involves not only top-down or passive, but also bottom-up or active emergence, negotiation and adaptation of meanings and practices (Feola 2014; Walsh-Russo 2014).

Issues of spatial versus cultural proximity have also been discussed in the growing body of scholarship on social movements and alternative media (e.g. Van Aelst and Walgrave 2002; Val Laer and Van Aelst 2010). Research in this area appears to agree that while in principle 'alternative' or 'new' media (e.g. internet, social media) facilitate less place-based and non-relational ties, and work on types of proximity other than the spatial, and therefore may influence diffusion towards less spatially structured patterns, in practice this is often not the case. The digital divide among countries (Norris 2001), the difficulty of establishing strong ties 'virtually' (Van Laer and Van Aelst 2010), the tendency of virtual networks to form, materialise and claim their agendas in particular places (Lim 2014), the actual and often overlooked geographical patterns of computer-mediated communication (Conover et al. 2013), and the difficulty of translating virtual connection into practical collective action (Diani 2000) are but a few of the reasons why the growing use of new media does not appear to result in less spatially structured spread, but rather in reinforced or new spatial structures of social movement diffusion.

In sum, it can be hypothesised that grassroots innovation diffusion is spatially structured, albeit in complex and unclear ways. Little work has been carried out so far on specific grassroots innovations for sustainability in a way that reveals their actual patterns of diffusion. While interpreting the geography 
of grassroots innovations entails more than understanding their spatial diffusion (Bridge et al. 2013), the latter remains important because the heterogeneity of grassroots innovations prevents us from formulating arguments about their emergence and development in different places (Ornetzeder and Rohracher 2013; Feola 2014; Feola and Nunes 2014).

\section{Methodology}

This study explored and compared the spatial diffusion of two networks of grassroots innovations in Great Britain (specifically England and Wales) and Italy. Specifically, this study addressed three questions on spatial diffusion. First, it asked whether grassroots innovation diffusion is spatially structured, that is, where grassroots innovations have diffused and whether they are randomly distributed in space. Second, this study investigated whether the spatial structure of grassroots innovation diffusion has changed over time. Third, and cutting across the previous two research questions, this study examined similarities and differences in the spatial diffusion of two different networks of grassroots innovations. The hypotheses initially formulated were that grassroots innovation diffusion would have a spatial structure. It was hypothesised that the two selected networks would show different spatial structures of diffusion, as they are characterised by different organisation, strategies and narratives (see sections below, and Feola 2014), and that the two branches of a single international movement (in Great Britain and Italy) would be characterised by similar spatial diffusion structures.

\section{Case studies}

Great Britain and Italy are relevant countries for this study because in both countries grassroots innovations have emerged strongly and acquired momentum in the last decades, and there are marked regional identities and economic differences (i.e. north-south divides) that may affect grassroots innovation diffusion. The specific grassroots innovations analysed were the Gruppi di Acquisto Solidale (Solidarity Purchasing Groups - GAS) in Italy, and The Transition Network (TTN) in Italy (TTN-IT) and Great Britain (TTN-GB). Both GAS and TTN are structured as networks, and the presence of TTN in both Great Britain and Italy allowed for a comparison of spatial diffusion between (i.e. TTN-GB and TTN-IT) and within (i.e. GAS and TTN-IT) countries.

Gruppi di Acquisto Solidale. Gruppi di Acquisto Solidale 'are groups of consumers who collectively manage direct purchasing (of food and non-food products, sometimes of services). Their size can range from some dozens to more than a hundred members' (Rossi and Brunori 2010, 1916). Following their initiation in 1994 in the city of Fidenza (Emilia Romagna region), GAS have witnessed rapid growth with 980 groups registered as of July 2014, although the number of GAS chapters may be higher since many groups remain informal.

GAS call into question existing economic structures and are often founded as critiques of consumerism (Colombo 2013). The groups or individuals joining GAS are usually motivated to make a change towards sustainability, and interpret consumption patterns as a powerful tool to advocate sustainability and bring about social and economic change. The groups set specific environmental and social justice criteria for the purchase of goods and services. In addition to ethical consumption, they encourage co-production and direct connection between producers and consumers (Rossi and Brunori 2010; Grasseni 2014). Thus, by promoting small, local, and ethical consumption, GAS aim to forge a more sustainable relationship between people, planet and profit (Colombo 2013).

GAS are autonomous in selecting producers and setting ethical and sustainability criteria, as well as ordering and distribution (Rossi and Brunori 2010). However, the GAS network plays a key role in distributing information about producers who comply with certain ethical standards (e.g. through the website and a mailing list). Regional or local networks of groups often connect to exchange information and experiences, or source products jointly, and in some cases inter-group coordination emerges (e.g. for the order of non-local products). Groups usually meet on a monthly basis, and the GAS network meets at national or regional levels on an annual basis (Rossi and Brunori 2010).

GAS have solid connections with other organisations, both at ideological and logistic levels. For example, distribution often occurs in structures made available by other social and political organisations (e.g. social centres, clubs, and churches), while GAS also 'have relationships with other local networks such as farmers' markets, small farmers' associations, fair trade organizations and social movements, with which they make joint initiatives' (Rossi and Brunori 2010, 1916).

GAS tend to diffuse through both relational (e.g. social networks) and non-relational (e.g. internet) pathways (Colombo 2013), and through an 'active seeker' model, as observed in solidarity economy movements elsewhere (Shawki 2013). Mediated diffusion pathways also appear to be in place, as ethical magazines, ethical businesses, solidarity economy fairs and events, and other civic movements play a role of brokering and translating GAS principles and practices into new environments and social circles. Diffusion also appears to occur through the division of groups that become too big to manage distribution or source products from a base of local producers. In these cases, a big group splits into 
two or more smaller groups with different location bases.

Transition Movement. The Transition Movement originated in Totnes, Devon, UK, in 2006 (Hopkins 2011) and has rapidly expanded to over 43 countries through more than 1100 local transition initiatives. The Transition Movement seeks to deal with the concomitant shrinkage of cheap fossil fuels, climate change, and economic crisis by creating resilient, cooperative and thriving communities. An 'energy descent' is often promoted through relocalisation and a series of initiatives that may include the redesign of food systems, transport, energy production, and the introduction of alternative currencies as means of local economic exchange.

The Transition Movement has developed a set of guidelines, originally modelled on the first transition initiative in Totnes and later updated and improved. These include a Transition Handbook (Hopkins 2008), a Transition Initiatives Primer (Brangwyn and Hopkins 2008) and Transition Companion (Hopkins 2011). Guidelines such as the 'transition model' (Brangwyn and Hopkins 2008) or the 'ingredients' of transition (Hopkins 2011) are meant to guide communities to set up successful local transition initiatives, but are often adapted by communities to their specific context (Shawki 2013). Thus, activist-adopters tend to be motivated by local issues, but look to the Transition Movement for inspiration (narratives, models of action), and see themselves as parts of a transnational movement (Shawki 2013). The Transition Network has also established a system of branding, according to which communities that desire to be recognised as official' members of the network need to comply with a set of criteria, including having attended a training session, having drafted and approved a constitution, being composed of at least four or five people, and demonstrating commitment to networking with others, including locally and with authorities (Brangwyn and Hopkins 2008). Transition initiatives that are inspired by the Transition Movement principles, but do not comply with these criteria, are listed as 'Muller' initiatives.

There is evidence that the diffusion of the Transition Movement involves relational (e.g. transition training, conferences), non-relational (e.g. books, website), and mediated diffusion (Bailey et al. 2010; Shawki 2013). Particularly, international, national and regional hubs of the Transition Network play a fundamental role in mediating the diffusion process by facilitating connections at particular scales and across scales (with the transnational movement) and facilitating social learning through training (Feola and Nunes 2014).

\section{Data collection and analysis}

Data related to the diffusion of GAS in Italy and TTN in Great Britain and Italy were collected from the databases available on the respective websites (i.e. retegas.org, transitionnetwork.org, transitionitalia .wordpress.com). These databases are sufficiently reliable sources of information, although they have some limitations due to the fact that (1) not all GAS and TTN groups register, and some do not so promptly after constitution; (2) the databases are usually updated only every few months; and (3) discontinued GAS or TTN groups may not be immediately removed from the databases. Where available, the websites, blogs or social media profiles of the GAS and TTN groups were visited to ascertain the activity status of the group. Both official and mulling transition initiatives (i.e. initiatives that are inspired by the Transition Movement principles, but that have not yet constituted themselves in the TTN) were considered in this study.

Important data for the study of diffusion are the foundation and termination dates of the solidarity purchasing groups and transition initiatives. These data were collected from the groups' websites, blogs or social media webpages, where they were often recorded in the groups' foundation documents or group profile webpage. When the information was not available, the registration date recorded on the GAS or TTN websites was used as a proxy for the groups' constitution dates.

GAS and TTN groups were geocoded and aggregated by spatial unit. The cartographic maps were acquired from the Italian and British statistical offices, respectively, and maps with comparable spatial units were used. These were provinces in Italy and local authority districts in Great Britain, whereby both spatial units are NUTS 3 level spatial units in the hierarchical system employed by the Statistical Office of the European Union. For Italy, where in recent years a number of new provinces have been created, the 2011 province boundaries were used. For Great Britain, the local authority district boundaries from 2012 were used. Data on the number of localities per province in Italy, and per local authority district in Great Britain, were collected from the respective statistical offices.

Data were analysed with GeoDa 1.4.6 (geodacenter.asu.edu). The exploratory spatial analysis was carried out in three major stages. First, the distribution of GAS, TTN-IT and TTN-GB (i.e. the number of GAS, TTN-IT and TTN-GB groups in each spatial unit) was mapped. However, as recommended by Anselin (2003), most of the analysis of diffusion patterns was based on the proportion (or percentage) of solidarity purchasing groups and transition initiatives in spatial units. The proportion was calculated as the ratio between the number of GAS, TTN-IT and TTN-GB and the number of localities in a given spatial unit. Therefore, the proportion gives a measure of the percentage of localities in a given spatial unit that feature at least one GAS, TTN-IT and TTN-GB, respectively. Quantile, standard deviation, 
and natural breaks maps were produced to identify outlier spatial units (i.e. those characterised by particularly high or low proportions).

Second, Moran's I statistic was used as a measure of global clustering to test the null hypothesis that the spatial structure of the data was random. A rejection of the null hypothesis (statistically significant Moran's $I$ ) implies a non-random spatial structure (spatial autocorrelation) (Anselin 2003). Two different ways of calculating adjacency, known as the Queen (which defines a location's neighbours as those with either a shared border or vertex) and Rook (defining a location's neighbours as those with a shared border only), were tested to define neighbour relationships, but no significant differences were observed. The analysis presented in this article refers to the Queen adjacency criterion (Anselin 2003).

Third, the local indicator of spatial autocorrelation (LISA) (Anselin 1995) was calculated to test whether the percentage of GAS and TTN groups for particular spatial units were closer to the values of a neighbouring unit or to the average of the study area (Anselin 1995). The statistical significance of LISA was determined by comparing the estimated LISA and a random reference distribution. A rejection of the null hypothesis (statistically significant LISA) implies a non-random spatial structure (i.e. local spatial autocorrelation), meaning the percentages of GAS and TTN groups correlate with values of neighbouring spatial units. The results of this analysis yielded five categories of spatial units, namely 'high-high', 'lowlow', 'high-low', 'low-high' and 'not significant'. These categories indicate clustering of adjusted proportions. High-high and low-low clusters indicate positive spatial autocorrelation, that is, clusters of spatial units characterised by significantly high or low proportions, respectively. High-low or low-high clusters indicate negative spatial autocorrelation, that is, clusters of spatial units with a high proportion spatial unit bordered by one or more low proportion spatial units, or a low proportion spatial unit bordered by one or more high proportion spatial units.

The varying size of spatial units (i.e. provinces in Italy and local authority districts in Great Britain) and the consequent population size (i.e. number of localities in each spatial unit) may result in population variance instability and affect the estimates. To minimise this potential issue, a spatial empirical Bayes rate smoothing approach was adopted (Anselin 2003). Both Moran's I and LISA were calculated for GAS and TTN for all the years of existence of these networks. Cluster maps were produced to visualise the findings.

\section{Results}

The spatial structure of grassroots innovations

GAS, TTN-IT and TTN-GB have grown in numbers over the last several years, whereby the growth of the two networks that have existed for longer periods of time (i.e. GAS and TTN-GB) has followed an S-shaped curve (Figure 1).

The growth of GAS, TTN-IT and TTN-GB has corresponded to a spatial diffusion that is not randomly distributed, and instead has a particular spatial structure. In Italy, GAS had diffused mostly in northern and central regions (Figure 2), and had a high proportion of local groups per localities in central Italy, and the Rome and Milan provinces (Figure 3). Similarly, TTN-IT is present mostly in provinces of central and north-eastern Italy (Figure 2). These provinces are also those in which the percentage of localities with at least one local transition initiative is highest (Figure 4). Moran's I for GAS (0.3598) and TTN-IT (0.3446) are both significant at a $1 \%$ level (Table 1$)$.

In Great Britain, TTN-GB is present in various spatial units and particularly in the south and the West Midlands (Figure 2). TTN-GB appears to have distributed more homogeneously than TTN-IT or GAS, as only in London and south England, and central Wales are there relatively higher and lower percentages, respectively, of localities with at least one local transition initiative (Figure 5).

\section{Change in the spatial structure of grassroots innovations over time}

The spatial structure of GAS diffusion has changed over time. In initial stages (years 2000-3), the provinces with the highest percentage of localities featuring at least one GAS group were those in the north-eastern part of the country. In a subsequent phase (2004-12), the highest percentages were in the provinces in central Italy. From 2012 to 2014, no substantial change to the spatial structure of GAS can be observed (Figure 6).

The spatial structure of TTN-IT diffusion does not appear to have substantially changed over time. This network originated in the provinces in central Italy, which have had the highest percentages of localities featuring at least one transition initiative. The cluster of provinces in central Italy with the highest proportions has enlarged over time (Figure 7). Interestingly, TTN-IT appears to have clustered around the same area in which it originated (Bologna province in the Emilia Romagna region), while GAS clusters have changed over time.

In Great Britain, the spatial structure of TTN-GB diffusion appears to have changed over time. In initial stages (years 2006-9), the local authority districts with the highest percentages of localities featuring at least one transition initiative were in the south-west (where TTN originated), the south and the Midlands. Subsequently (2010-14), local authority districts in the south-west do not appear to be among those with highest percentage of localities with at least one transition initiative (Figure 8), although they 

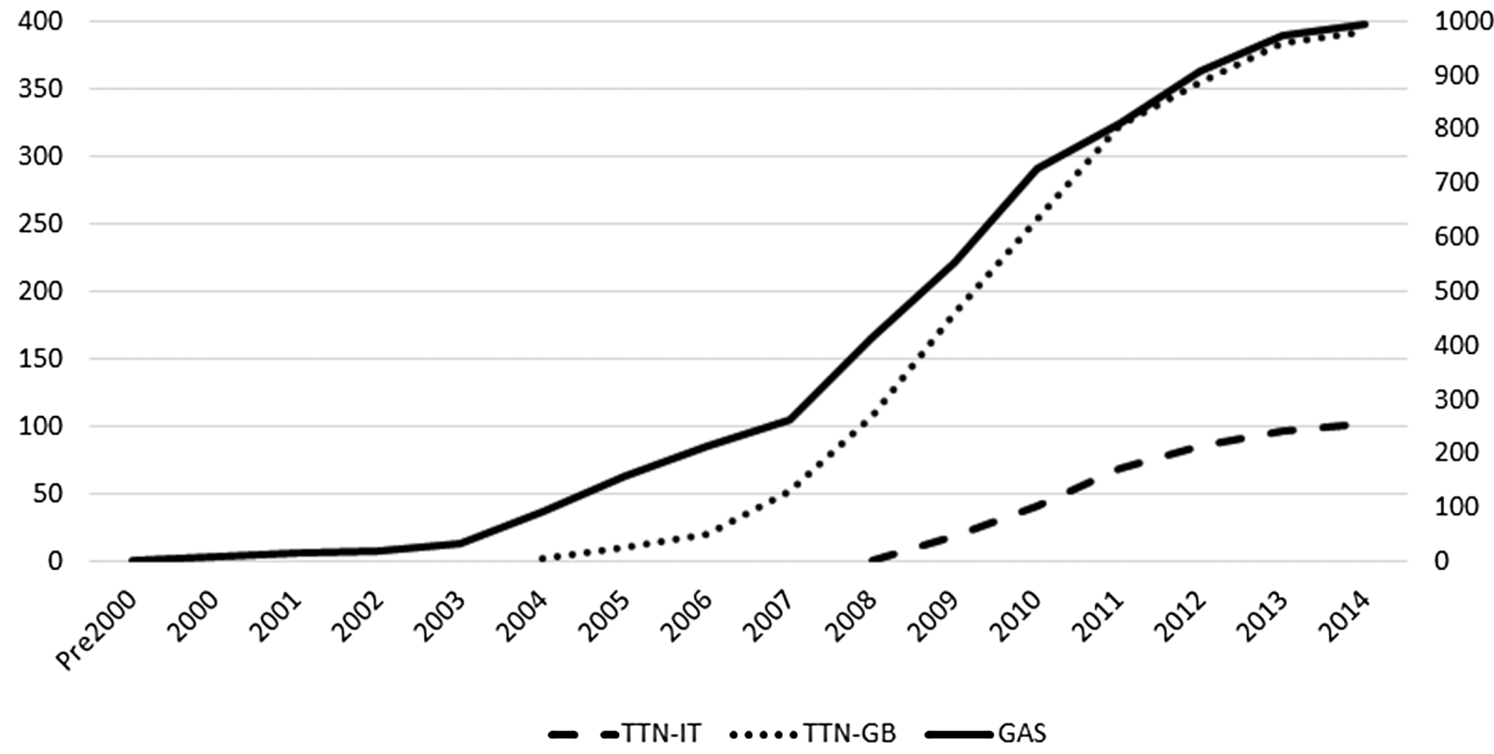

Figure 1 Number of solidarity purchasing groups (GAS), transition initiatives in Italy (TTN-IT) and Great Britain (England and Wales) (TTN-GB) (years 2000-14)

continued to play host to a large number of transition initiatives (Figure 2).

Thus, for both GAS and TTN-GB, changes in the spatial structure of GAS over time coincided with moments of substantial growth of the networks (Figure 1). This suggests that the steeper increases in numbers registered over these periods have occurred more than proportionally in central Italy (GAS) and the northern and eastern districts of England and Wales (TTN-UK) than in other parts of the countries. In other words, the diffusion of these grassroots innovation networks has been spatially uneven, and some provinces and local authority districts have driven the growth in numbers of GAS and TTN-GB more than others. In these spatial units, a high number of localities have seen the emergence of GAS and TTN-GB groups. It is not possible to compare the spatial diffusion of TTN-IT over time to the other two networks as TTN-IT has had a shorter existence.

Finally, it can be observed that Moran's / for GAS and TTN-IT has increased in value and significance over time (Table 1). A similar trend, albeit at lower values of Moran's I and significance levels, can be observed for TTN-UK (Table 1).

In sum, the comparison of spatial patterns of diffusion over time within and across countries confirmed that GAS and TTN diffusion in Italy and Great Britain is spatially structured, and that the spatial structure of diffusion has changed over time, although more clearly in the case of GAS and TTN-IT than of TTN-GB. The analysis also suggests that despite belonging to the same transnational movement, TTN-IT and TTN-GB have diffused differently in different countries. TTN-IT has largely adopted the TTN and TTN-GB principles and practices, but these two grassroots networks have diffused, resulting in distinct spatial diffusion patterns (i.e. clearly clustered in Italy, less so in England and Wales). This difference does not appear to depend on the different stage of development of TTN-IT and TTNGB, as TTN-GB did not show in the early stages of diffusion such levels of clustering that characterises TTN-IT. On the other hand, GAS and TTN-IT differ regarding principles, activities and organisational practices, but have diffused in the same parts of the country, namely the centre and north-east, and have more densely concentrated in some provinces, notably in central Italy.

\section{Discussion and conclusions}

A growing body of scholarship has highlighted issues of scale, place and space in relation to grassroots innovation. By looking at broader spatial diffusion patterns, this study contributes to that scholarship and offers further insights towards the understanding of why grassroots innovations diffuse in some places and not in others and, consequently, how they can be 
$\mathrm{GAS}^{*}$

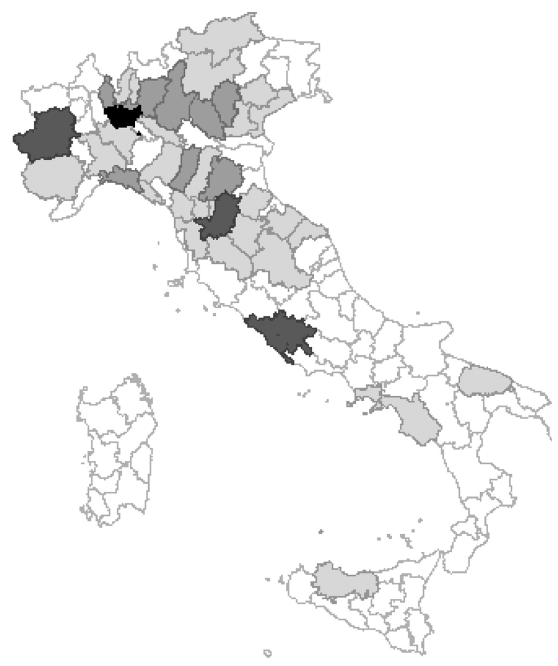

TTN-GB***

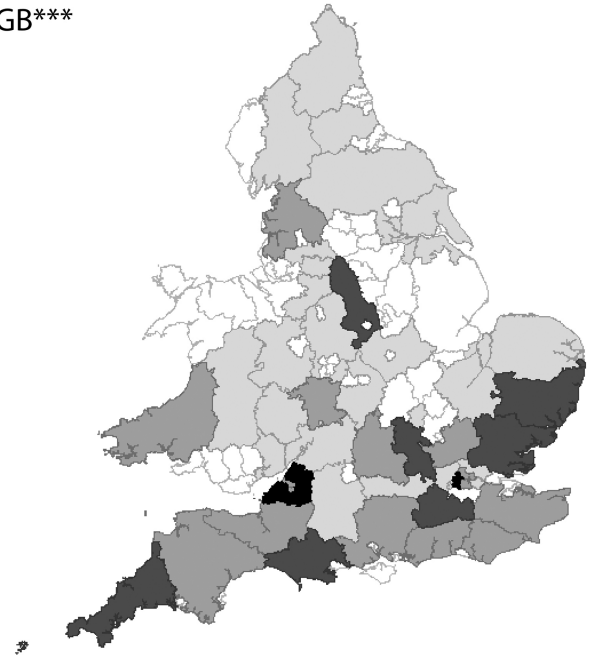

TTN-IT**

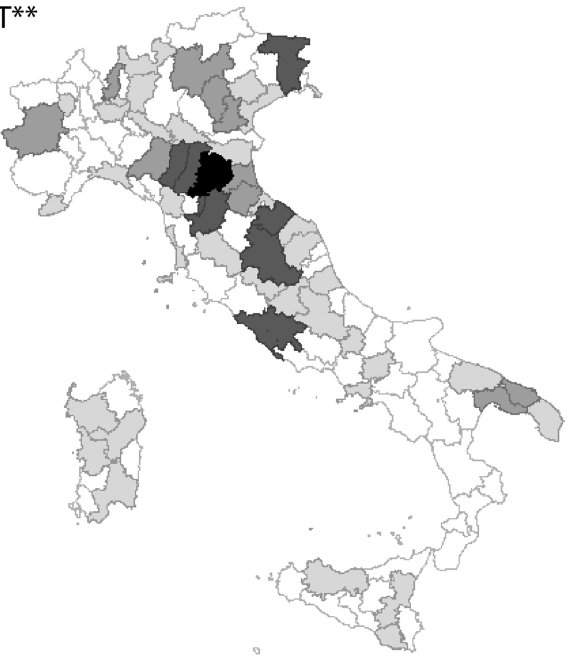

Figure 2 Location of solidarity purchasing groups and transition initiatives in Italy and Great Britain (England and Wales) (TTN-GB) (year 2014)

*White: 0-6 groups (69 provinces); light grey: 7-16 groups (28 provinces); medium grey: 19-19 groups (9 provinces); dark grey: $53-75$ groups ( 3 provinces); black: 99 groups ( 1 province). ${ }^{* *}$ White: 0 transition initiatives (56 provinces); light grey: 1 transition initiative (35 provinces); medium grey: 2 transition initiatives (10 provinces); dark grey: $3-4$ transition initiatives (8 provinces); black: 21 transition initiatives ( 1 province). ${ }^{* * *}$ White: $0-2$ transition initiatives (64 districts); light grey: 3-5 transition initiative (25 districts); medium grey: 6-9 transition initiatives (13 districts); dark grey: 10-14 transition initiatives (7 districts); black: 20-28 transition initiatives (2 districts) 
Year 2003

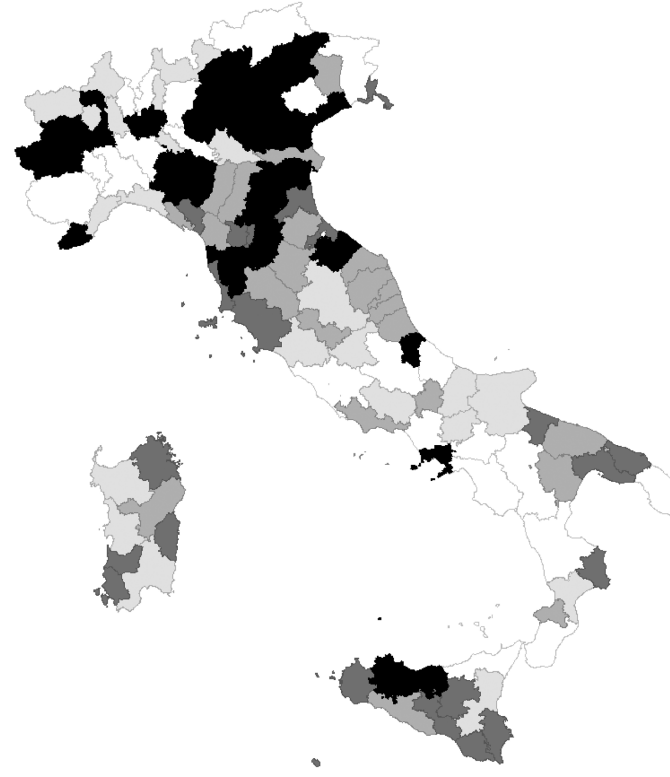

Year 2010

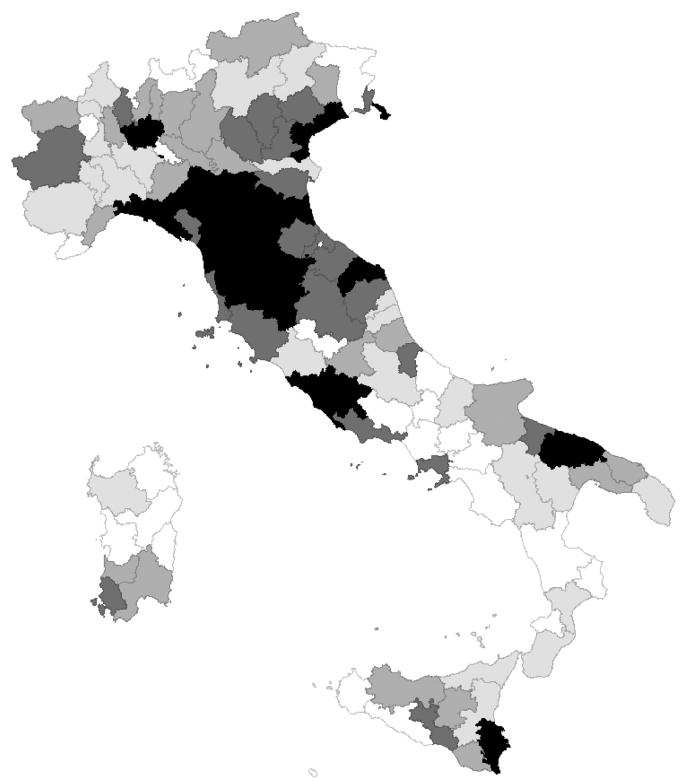

Year 2007

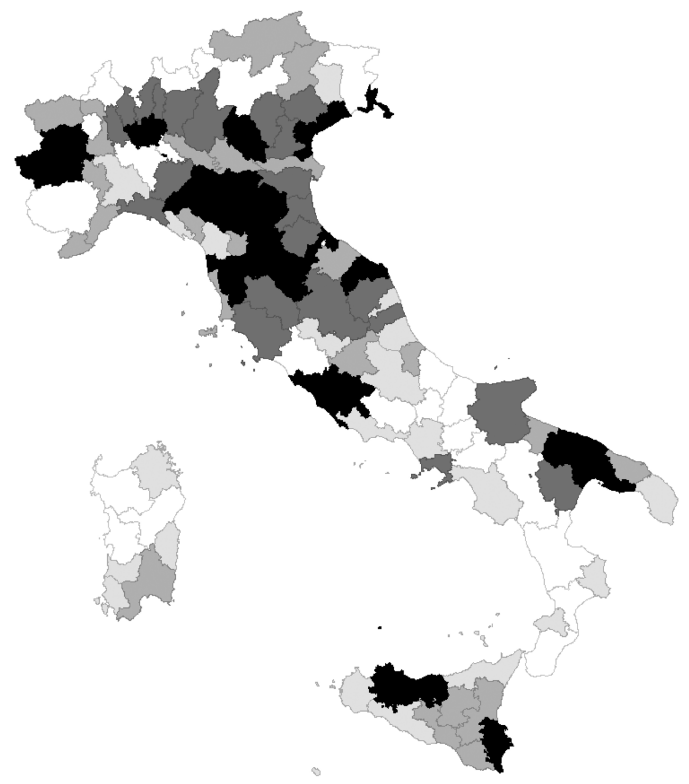

Year 2014

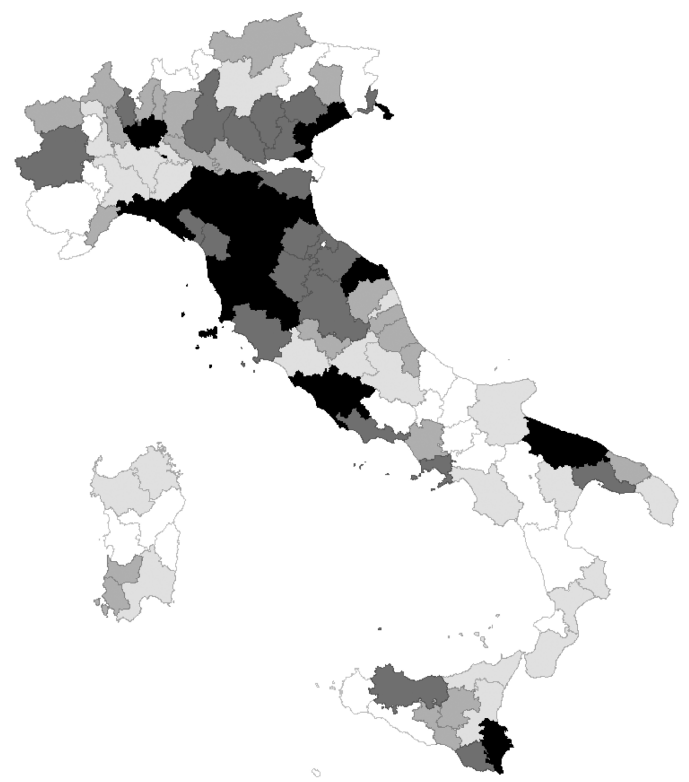

Figure 3 Proportion of local solidarity purchasing groups (GAS) in selected years (empirical Bayes-smoothed) Darker colours denote spatial units in which a higher number of localities feature at least one GAS. White: first quintile; light grey: second quintile; medium grey: third quintile; dark grey: fourth quintile; black: fifth quintile 


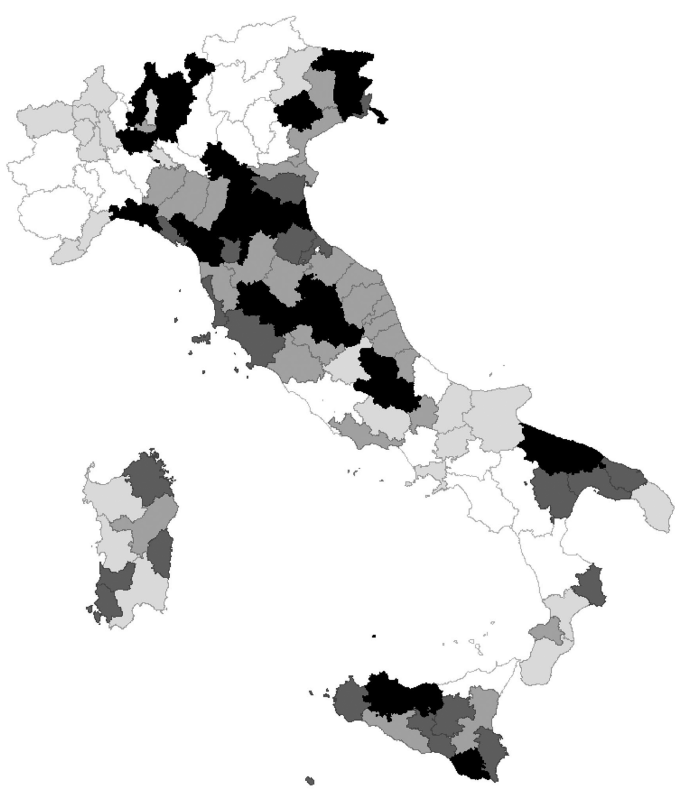

Year 2012

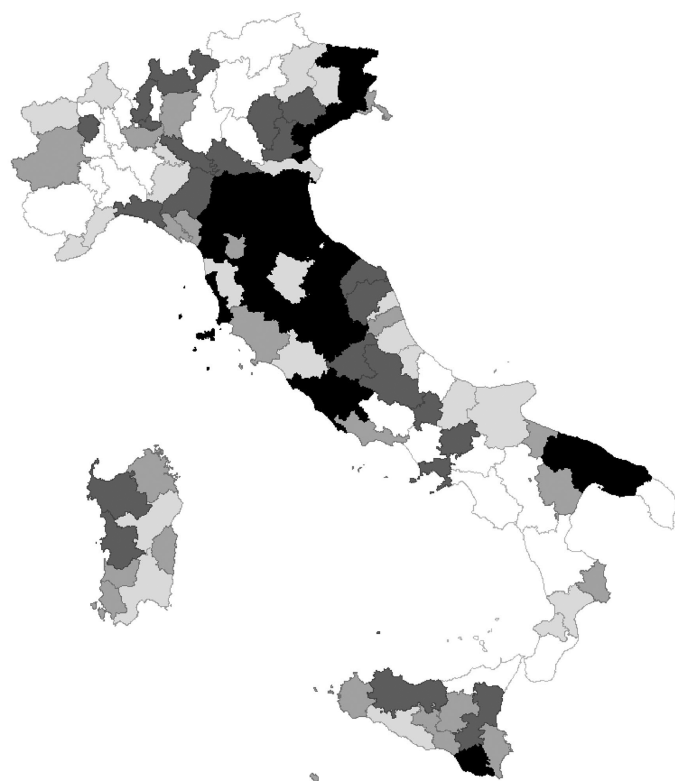

Year 2011

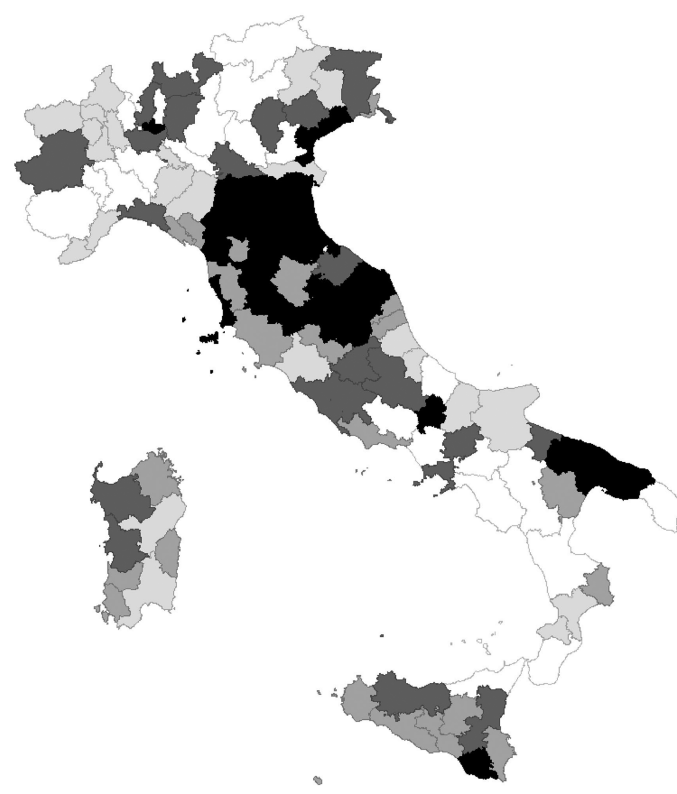

Year 2014

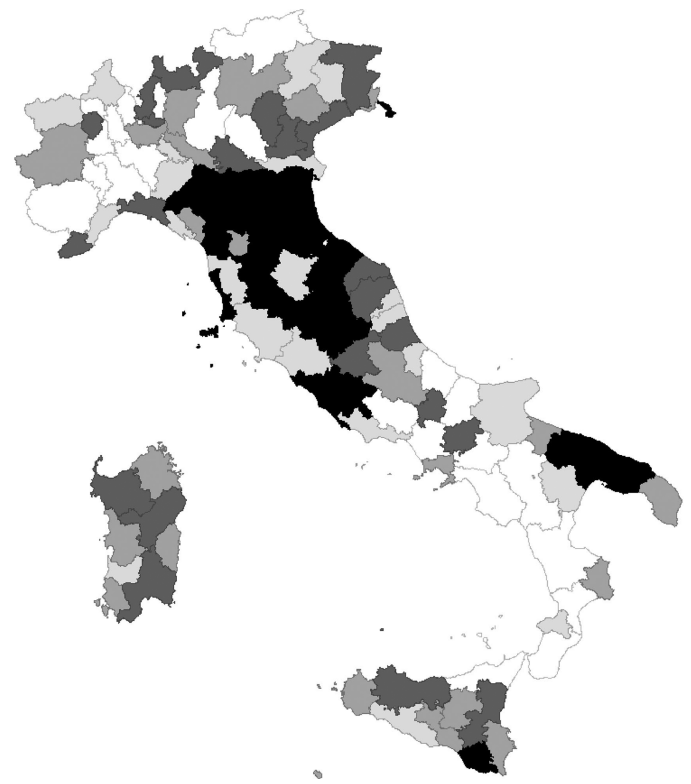

Figure 4 Proportion of transition initiatives in Italy in selected years (empirical Bayes-smoothed) Darker colours denote spatial units in which a higher number of localities feature at least one transition initiative. White: first quintile; light grey: second quintile; medium grey: third quintile; dark grey: fourth quintile; black: fifth quintile 
Table 1 Spatial autocorrelation of empirical Bayes-adjusted rates of solidarity purchasing groups (GAS), transition initiatives in Italy (TTN-IT) and Great Britain (England and Wales) (TTN-GB). Moran's I values and significance (years 2000-14)

\begin{tabular}{lccc}
\hline Year & GAS & TTN-IT & TTN-GB \\
\hline 2000 & 0.0962 & & \\
2001 & 0.0180 & & \\
2002 & -0.0309 & & \\
2003 & -0.0002 & & \\
2004 & 0.0574 & & -0.5000 \\
2005 & $0.1263^{*}$ & & $0.0720^{*}$ \\
2006 & $0.1212^{*}$ & & $0.0358^{*}$ \\
2007 & $0.1610^{*}$ & & $0.0826^{*}$ \\
2008 & $0.3268^{* *}$ & -0.0050 & $0.1385^{*}$ \\
2009 & $0.3274^{* *}$ & $0.0855^{*}$ & $0.1090^{*}$ \\
2010 & $0.3332^{* *}$ & $0.2806^{* *}$ & $0.2068^{* *}$ \\
2011 & $0.3166^{* *}$ & $0.3299^{* *}$ & $0.2388^{*}$ \\
2012 & $0.3308^{* *}$ & $0.3449^{* *}$ & $0.2157^{*}$ \\
2013 & $0.3572^{* *}$ & $0.3586^{* *}$ & $0.2027^{*}$ \\
2014 & $0.3598^{* *}$ & $0.3446^{* *}$ & \\
\hline
\end{tabular}

Higher Moran's I values denote stronger overall clustering in the respective datasets. *Significant at $10 \%$ level; **significant at $1 \%$ level

encouraged and supported. Key to this goal is understanding how place-based factors interact with transnational repertoires of practices, and how different types of proximities (geographical as well as institutional, social, cognitive and organisational) interact with and influence one another. Thus, this study's findings can be interpreted along two interrelated lines of analysis, which mirror two major foci of current literature on the geography of grassroots innovations for sustainability: place-based conditions for the emergence and success of grassroots innovations, and the mechanisms for their diffusion.

Recent literature has highlighted the importance of favourable place-based and unevenly distributed conditions for the emergence and success of grassroots movements (e.g. Pickerill and Maxey 2009; Ornetzeder and Rohracher 2013; Feola and Nunes 2014). As argued by Andrews and Biggs (2006), local conditions, such as the presence of a particular population profile or favourable political conditions, can play an important role in spatially structuring the diffusion of social movements. Particularly, North (2010b) and Shawki (2013) further supported this by each showing the importance of a concentration of like-minded people and active seekers of innovation for the longevity of grassroots innovations. These forms of cultural proximity often intersect social and organisational structures (e.g. social networks, forms of organisation) (Boschma 2005). These pre-existing place-based conditions may be encountered in selected regions in Italy and Great Britain and explain why in these countries, the diffusion of TTN and GAS is spatially structured. For example, central Italy is historically a politically left-wing area, and is characterised by a deeply rooted network of social enterprises, cooperatives and associations and relatively high levels of environmental awareness among the population and the political elite. The northeast, often referred to as the 'white region', has a longstanding presence of a network of Catholic associations and activists (Putnam et al. 1994). Not surprisingly, one of the most iconic examples of local authority support of a TTN-IT initiative is that of the town of Monteveglio in the Emilia Romagna Region (Hopkins 2011), which was one of the first of this kind. There is debate over whether local government support for grassroots innovations influences or impedes their development (e.g. Jonas 2010; Feola and Nunes 2014). Nevertheless, the example of Monteveglio illustrates how in these regions there is not only a more widespread acceptance of the environmental threats, a higher proportion of likeminded peoples and pre-existing social and organisation structures (Boschma 2005; North 2010b; Shawki 2013), but, importantly, gatekeepers (i.e. politicians) and leaders who may play a substantial supporting role (Bailey et al. 2010). This may have favoured the emergence and diffusion of grassroots innovations in these geographical areas.

In England, Bailey et al. (2010) pointed out that TTN-UK emerged in small towns with strong social networks characterised by a 'progressive' culture in the southwest of England (see Figures 2 and 5). However, North and Longhurst (2013) argued that while TTN-UK had initially emerged in small towns, urban centres can be fertile grounds for TTN 'because [they allow] for a greater diversity of political action as well as providing a density of networks and resources that can be critical for the survival of grassroots interventions' (p. 1423). Furthermore, London is traditionally a pole of attraction and engine of numerous dynamics in the political, cultural, social, and economic spheres (Huggins 2003). The potential for urban areas (e.g. London, but also other cities like Bristol) to host TTN-UK initiatives is confirmed by this study, although the limited statistical significance of the spatial structure in Great Britain (Table 1) suggests that TTN-UK may diffuse in small towns and larger urban centres alike.

A second line of analysis to which this study's findings connect is that of the mechanisms of diffusion. The literature has shown that GAS tend to diffuse through both relational (e.g. social networks) and non-relational (e.g. internet) pathways (Colombo 2013), and through an 'active seeker' model, as observed for solidarity economy movements elsewhere (Shawki 2013). The diffusion of TTN involves relational (e.g. transition training, 

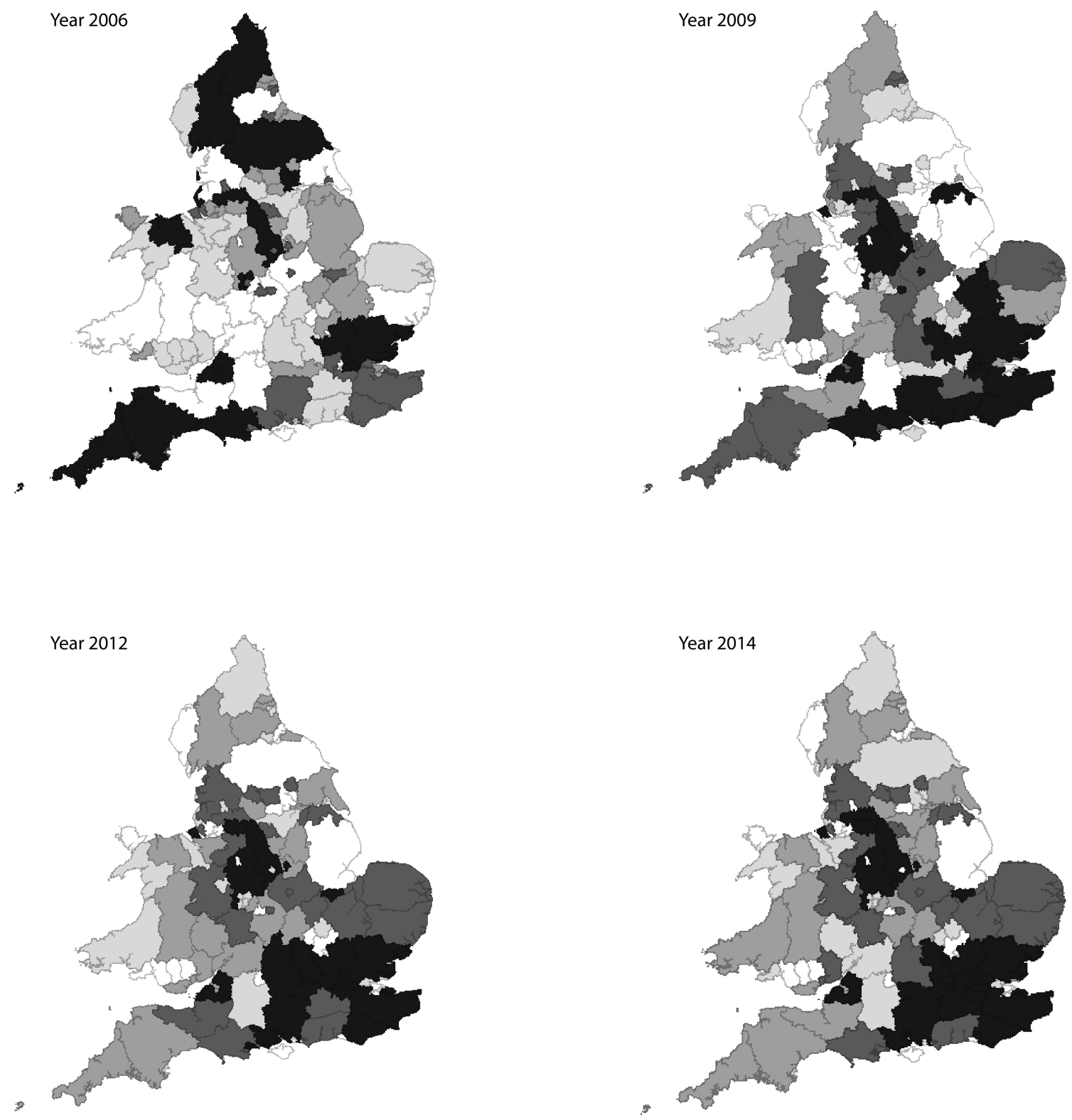

Figure 5 Proportion of transition initiatives in Great Britain (England and Wales) in selected years (empirical Bayes-smoothed)

Darker colours denote spatial units in which a higher number of localities feature at least one transition initiative. White: first quintile; light grey: second quintile; medium grey: third quintile; dark grey: fourth quintile; black: fifth quintile 
Year 2003

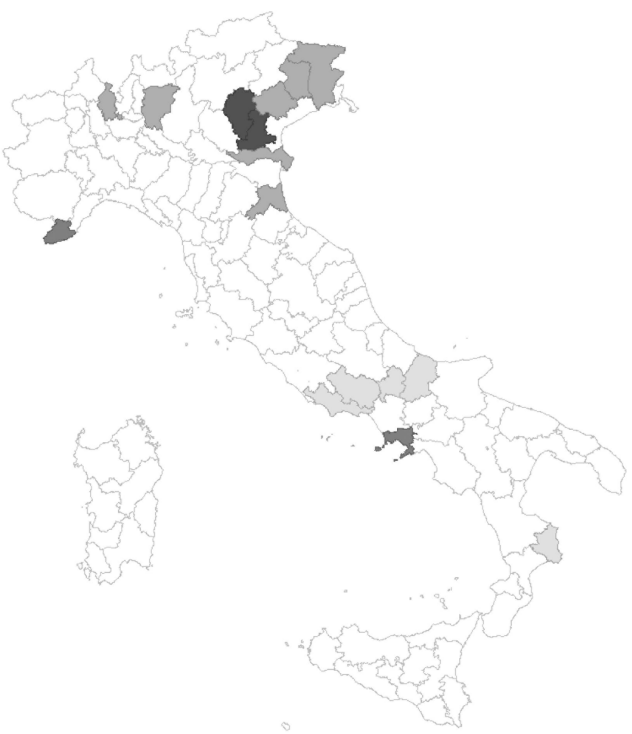

Year 2010

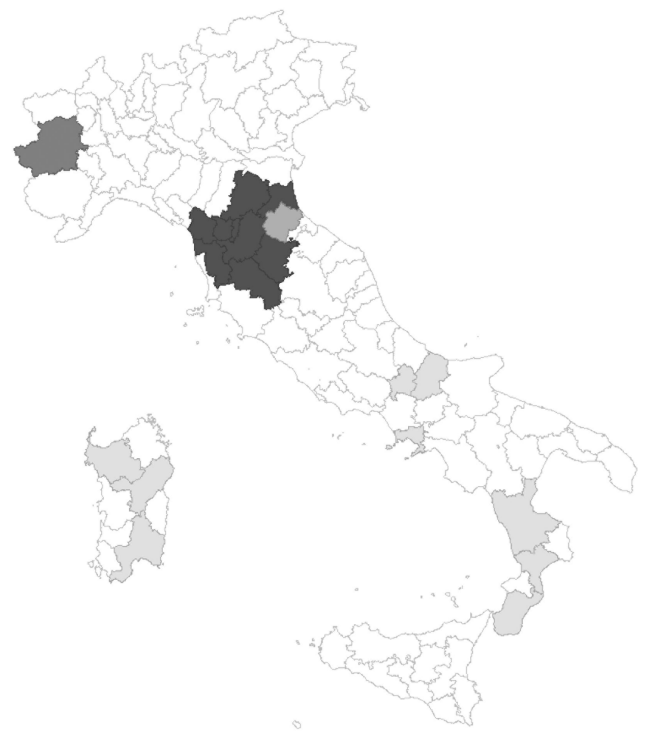

Year 2007

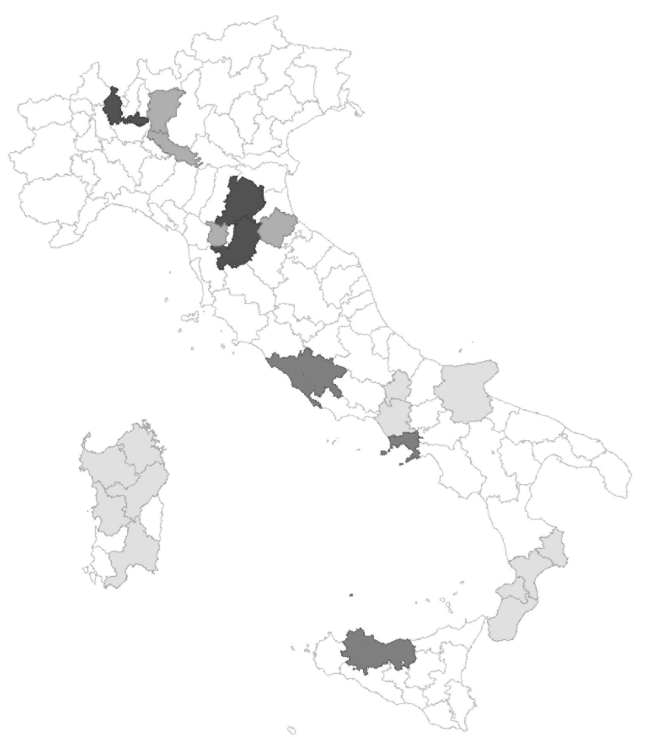

Year 2014

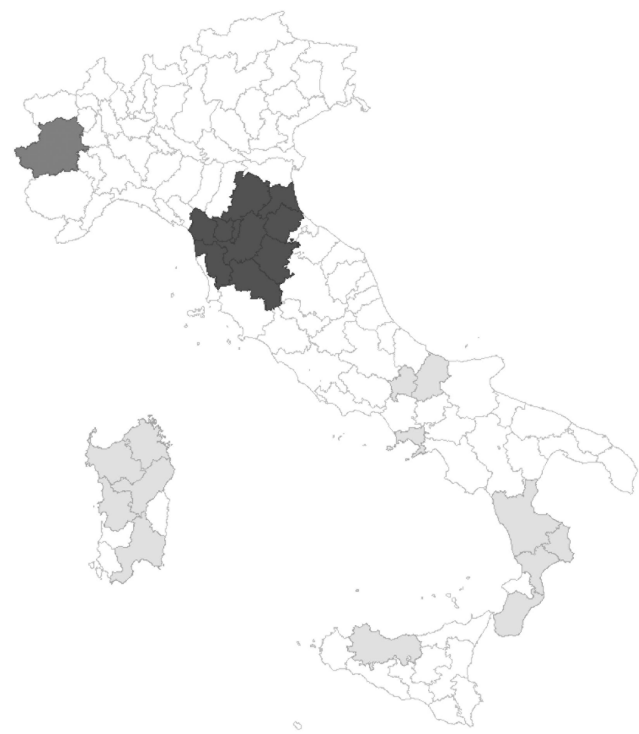

Figure 6 Local indicator of spatial autocorrelation (empirical Bayes-smoothed) for solidarity purchasing groups in Italy (selected years)

Colours denote spatial units whose proportions of GAS and TTN groups correlate with values of neighbouring spatial units. White: non-significant; black: high-high cluster; dark grey: high-low cluster; medium grey: low-high cluster; light grey: low-low cluster 
Year 2009

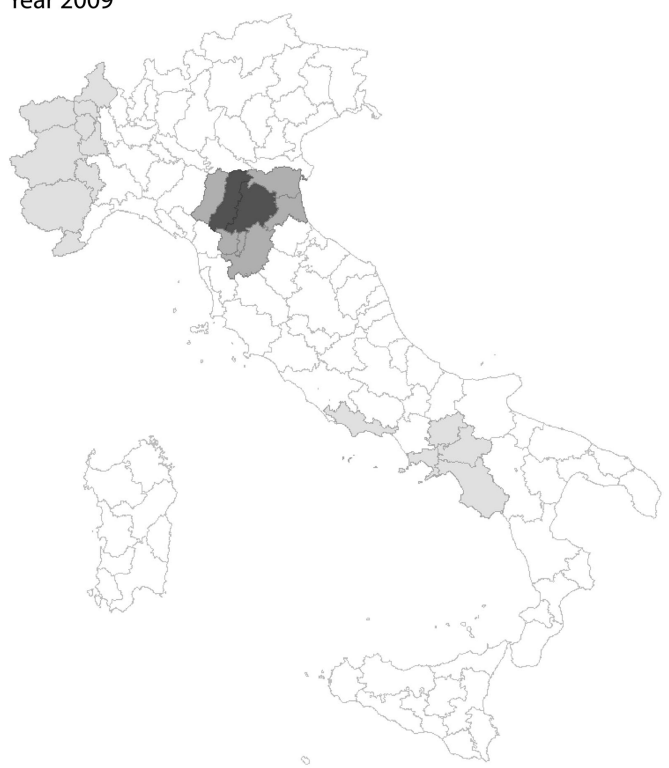

Year 2012

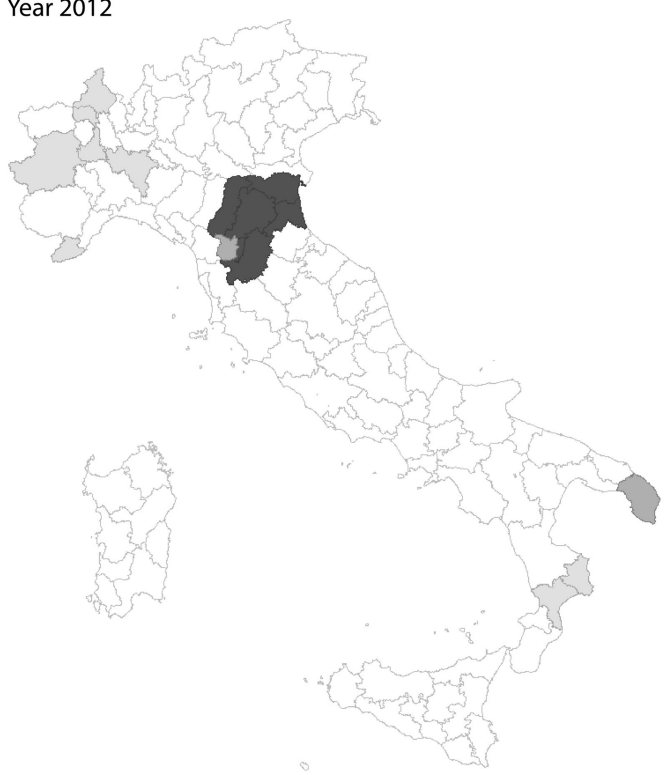

Year 2011

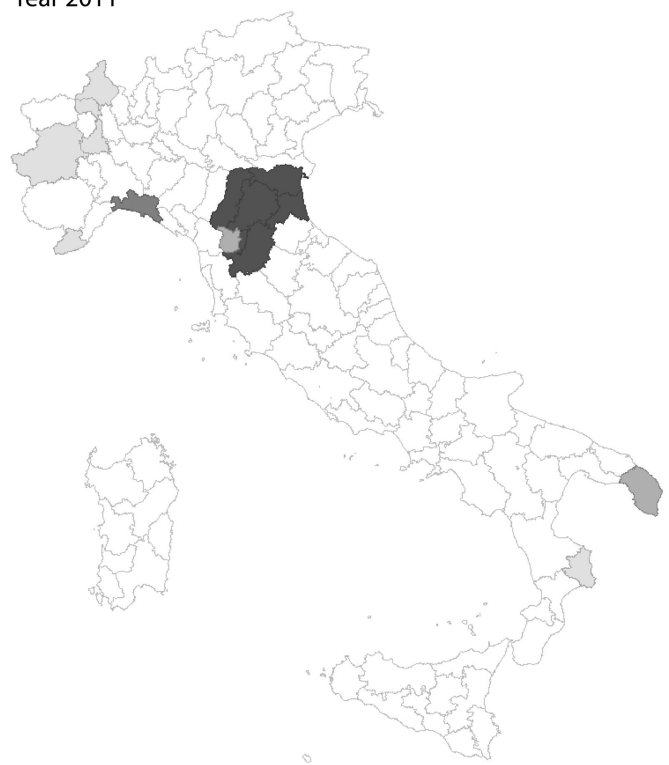

Year 2014

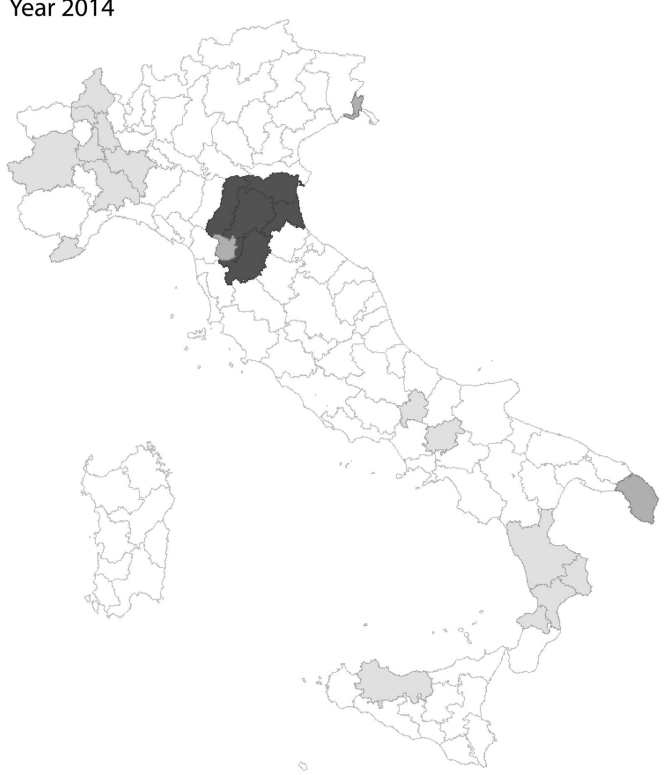

Figure 7 Local indicator of spatial autocorrelation (empirical Bayes-smoothed) for transition initiatives in Italy (selected years)

Colours denote spatial units whose proportions of GAS and TTN groups correlate with values of neighbouring spatial units. White: non-significant; black: high-high cluster; dark grey: high-low cluster; medium grey: low-high cluster; light grey: low-low cluster 

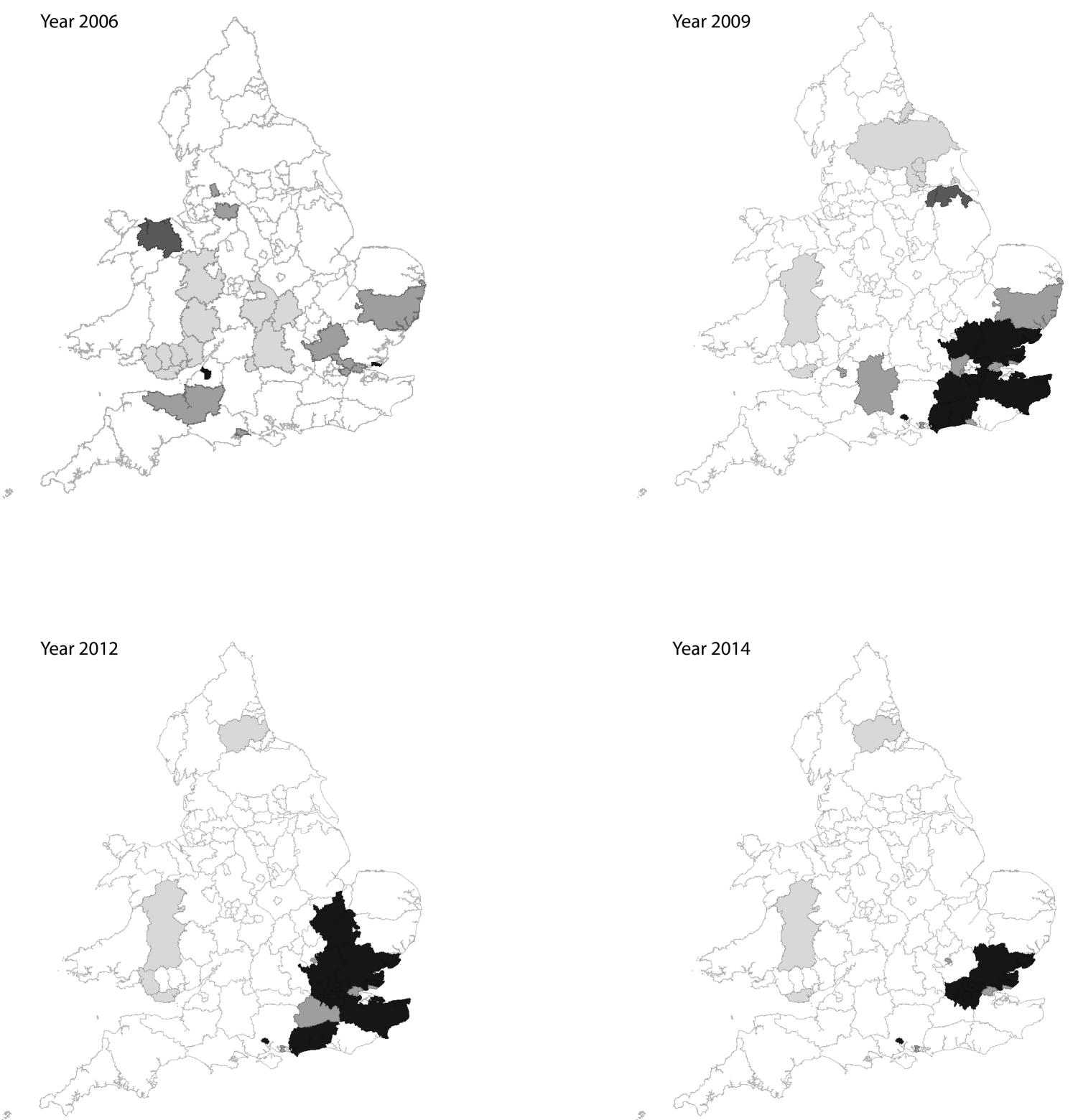

Figure 8 Local indicator of spatial autocorrelation (empirical Bayes-smoothed) for transition initiatives in Great Britain (England and Wales) (selected years)

Colours denote spatial units whose proportions of GAS and TTN groups correlate with values of neighbouring spatial units. White: non-significant or borderless; black: high-high cluster; dark grey: high-low cluster; medium grey: low-high cluster; light grey: low-low cluster. 
conferences), non-relational (e.g. books, website), and mediated diffusion (Bailey et al. 2010, Shawki 2013).

By uncovering the similarities and differences among grassroots movements, this study sheds further light on possible diffusion mechanisms. Distinct grassroots networks like GAS and TTN-IT, despite differences in their organisational practices and ideological basis, have diffused in the same parts of the country, namely the centre and northeast, and have concentrated in some provinces in central Italy (Figures 2-4). Local transfers of practices and strategies occurring between movements operating in a given place may have contributed to GAS and TTN-IT diffusion, which is reflected in similar spatial structures of diffusion of different grassroots innovations.

McAdam and Rucht (1993) argued that social movements should not be considered in isolation, in that there is often a transfer of elements across similar groups. Third-sector events such as fair trade or ethical finance fairs, or third-sector conferences, which have seen a growing popularity in Italy over the last few years, may have served this function (Colombo 2013; Grasseni 2014). These events may not only favour relational diffusion (Shawki 2013), but fertilisation across networks. This is plausible, since such events have taken place mostly in north and central Italy, where both GAS and TTN-IT have tended to cluster. Similarly in the UK, TTN's international conference takes place in London or the southwest and attracts mostly participants from Great Britain, which possibly reinforces the spatial distribution of this network in these geographical areas. Still, GAS and TTN appear to retain differences in principles, activities and organisational practices (see above, and Feola 2014), and therefore it is unclear to what extent, and regarding what practices, such a transfer may be occurring. As noted by Bailey et al. (2010), it is uncertain how TTN's strategy of rhizomic networking can penetrate other networks with similar forms of propagation.

It is also possible that different but proximate movements such as TTN-IT and GAS are actively connected in collaborations across networks at the local or regional level, such as in Solidarity Economy Districts that have recently emerged in Italy (Grasseni 2014), which results in similar spatial diffusion structures. While the actual, operational connections of GAS and TTN-IT were not tested in this study, the spatial patterns of diffusion uncovered in this study suggest that both Solidarity Economy Districts and the above-mentioned third-sector events may be playing a role in the diffusion of these networks, thereby illustrating the importance of territorially intensive relations (Nicholls 2007) and locally embedded forms of knowledge (Ornetzeder and Rohracher 2013).

To conclude, this study sheds new light on the common narrative that presents these networks as spreading virally or in a rhizome-like fashion. These metaphors are often used to emphasise the momentum of grassroots innovation diffusion, and the spaces of possibility for alternatives to emerge in the interstices of mainstream, neoliberal economies (Hopkins 2009; Pickerill and Maxey 2009; Bailey et al. 2010; Scott-Cato and Hillier 2010). The rhizome metaphor emphasises networking, relationality and transversality rather than spatial proximity, and aleatory connectivity rather than patterns and structure in the process of diffusion. In fact, this study indicates that grassroots innovation diffusion in space is structured and suggests that such spatial structure may depend on place-specific conditions and diffusion mechanisms. Importantly, this study also showed that spatial patterns of diffusion may change over time. Insofar as grassroots innovations can be agents of change towards sustainability, it is important to understand why this change occurs, and whether this reflects modifications in grassroots movement strategies (e.g. more or less use of the internet for communication, learning and recruitment), mechanisms of diffusion (e.g. interaction among emergent and existing movement networks), or in the contextual conditions (e.g. political support). Changes in spatial diffusion patterns, therefore, may highlight moments of rupture in particular places while absence may indicate lock-in situations into particular pathways (Wilson 2012; Bridge et al 2013). Spatial analysis can help identify pathways of transition to sustainability in different places, and thereby help advance our understanding of the geography of grassroots innovations for sustainability by complementing both individual case studies and studies focusing on diffusion mechanisms, which have characterised transition and social movement studies, respectively. To comprehend whether and to what extent the spaces of diffusion of grassroots innovations represent spaces of radical and alternative innovation for sustainability, how they come to exist, and what characterises them, is important to support the emergence and diffusion of alternatives to the unsustainable economies of neoliberal capitalism.

\section{Acknowledgments}

The authors wish to thank Emanuele Greco for his contribution to the data collection, Michael Goodman and Richard Nunes for their very useful comments on an earlier version of this manuscript, and Annalisa Feola for her help with the production of the figures. The authors are also thankful to the University of Reading's UROP scheme and School of Archaeology, Geography and Environmental Science for funding the study.

\section{References}

Andrews K T and Biggs M 2006 The dynamics of protest diffusion: movement organizations, social networks, and news 
media in the 1960 sit-ins American Sociological Review 71 752-77

Anselin L 1995 Local indicators of spatial association - LISA Geographical Analysis 27 93-115

Anselin L $2003 \mathrm{GeoDa}^{\mathrm{TM}} 0.9$ user's guide Spatial Analysis Laboratory, University of Illinois, Urbana-Champaign, and Center for Spatially Integrated Social Science, University of Santa Barbara

Balland P-A, Boschma R and Frenken K 2014 Proximity and innovation: from statics to dynamics Regional Studies 1-14

Bailey I, Hopkins R and Wilson G 2010 Some things old, some things new: the spatial representations and politics of change of the peak oil relocalisation movement Geoforum 41595 605

Boschma R 2005 Proximity and innovation: a critical assessment Regional Studies 39 61-74

Brangwyn B and Hopkins R 2008 Transition initiatives primer: becoming a transition town, city, district, village, community or even island (www.transitionnetwork.org/sites/default/files/ TransitionInitiativesPrimer\%283\%29.pdf)

Bridge G, Bouzarovski S, Bradshaw M and Eyre N 2013 Geographies of energy transition: space, place and the lowcarbon economy Energy Policy 53 331-40

Caprotti F and Bailey I 2014 Making sense of the green economy Geografiska Annaler: Series B Human Geography 96 195-200

Coenen L, Benneworth P and Truffer B 2012 Toward a spatial perspective on sustainability transitions Research Policy 41 968-79

Colombo L 2013 The gas as laboratories of civil economy

Conover M D, Davis C, Ferrara E, McKelvey K, Menczer F and Flammini A 2013 The geospatial characteristics of a social movement communication network PloS One 8 e55957

Diani M 2000 Social movements virtual and real Information, Communication \& Society 3 386-401

Feola G 2014 Narratives of grassroots innovations: a comparison of Voluntary Simplicity and the Transition Movement in Italy International Journal of Innovation and Sustainable Development 8 250-69

Feola G and Nunes R 2014 Success and failure of grassroots innovations for addressing climate change: the case of the Transition Movement Global Environmental Change 24 232-50

Fudge S, Jackson T and Peters M 2010 Low carbon communities. Imaginative approaches to combating climate change locally Edward Elgar, Basingstoke

Gibbs D and O'Neill K 2014 The green economy, sustainability transitions and transition regions: a case study of Boston Geografiska Annaler: Series B, Human Geography 96 201-16

Grasseni C 2014 Seeds of trust. Italy's Gruppi di Acquisto Solidale (Solidarity Purchase Groups) Journal of Political Ecology 21 178-92

Hedström P 1994 Contagious collectivities: on the spatial diffusion of Swedish trade unions, 1890-1940 American Journal of Sociology 99 1157-79

Hopkins R 2008 The transition handbook: from oil dependency to local resilience Green Books, Totnes

Hopkins R 2009 Transition to a world without oil TED Global Conference, Oxford

Hopkins R 2011 The transition companion Green Books, Totnes
Huggins R 2003 Creating a UK competitiveness index: regional and local benchmarking Regional Studies 37 89-96

Jonas A E G 2010 'Alternative' this, 'alternative' that ... interrogating alterity and diversity in Duncan F, Jonas A E G and Lee $\mathbf{R}$ eds Interrogatin alterity: alternative economic and political spaces Ashgate, Farnham 3-30

Leach M, Rockström, J, Raskin P, Scoones I, Stirling A C, Smith A, Thompson J, Millstone E, Ely A, Arond E, Folke C and Olsson P 2012 Transforming innovation for sustainability Ecology and Society 1711

Lim M 2014 Seeing spatially: people, networks and movements in digital and urban spaces International Development and Planning Review 36 51-72

Markard J, Raven R and Truffer B 2012 Sustainability transitions: an emerging field of research and its prospects Research Policy 41 955-67

McAdam D and Rucht D 1993 The cross-national diffusion of movement ideas Annals of the American Academy of Political and Social Science 528 56-74

McAdam D, Tarrow S and Tilly C 2001 Dynamics of contention Cambridge University Press, Cambridge

Newton P and Newman P 2013 The geography of solar photovoltaics (PV) and a new low carbon urban transition theory Sustainability 5 2537-56

Nicholls W J 2007 The geographies of social movements Geography Compass 1 607-22

Norris P 2001 Digital divide: civic engagement, information poverty, and the Internet worldwide Cambridge University Press, Cambridge

North P 2010a Eco-localisation as a progressive response to peak oil and climate change - A sympathetic critique Geoforum 41 585-594

North P 2010b The longevity of alternative economic practices: lessons from alternative currency networks in Duncan F, Jonas A E G and Lee R eds Interrogatin alterity: alternative economic and political spaces Ashgate, Farnham $31-46$

North P and Longhurst N 2013 Grassroots localisation? The scalar potential of and limits of the 'transition' approach to climate change and resource constraint Urban Studies 50 1423-38

Ornetzeder M and Rohracher H 2013 Of solar collectors, wind power, and car sharing: comparing and understanding successful cases of grassroots innovations Global Environmental Change 23 856-67

Peters M, Fudge S and Jackson T eds 2010 Low carbon communities: imaginative approaches to combating climate change locally Edward Elgar Publishing, Cheltenham

Pickerill J and Maxey L 2009 Geographies of sustainability: low impact developments and radical spaces of innovation Geography Compass 3 1515-39

Putnam R D, Leonardi R and Nanetti R Y 1994 Making democracy work: civic traditions in modern Italy Princeton University Press, Princeton NJ

Raven R, Schot J and Berkhout F 2012 Space and scale in socio-technical transitions Environmental Innovation and Societal Transitions 4 63-78

Rossi A and Brunori G 2010 Drivers of transformation in the agro-food system. GAS as co-production of Alternative Food 
Networks. Building sustainable rural futures. The added value of systems approaches in times of change and uncertainty in Darnhofer I and Grötzer $\mathbf{M}$ eds Proceedings of the 9th European IFSA Symposium University of Natural Resources and Applied Life Sciences, Vienna

Schulz C and Bailey I 2014 The spatial dimensions of the green economy and post-growth regimes: opportunities and challenges for economic geography Geografiska Annaler B 96 277-91

Scott-Cato M and Hillier J 2010 How could we study climaterelated social innovation? Applying Deleuzean philosophy to transition towns Environmental Politics 19 869-87

Seyfang G 2009 The new economics of sustainable consumption: seeds of change Palgrave Macmillan, London

Seyfang G and Smith A 2007 Grassroots innovations for sustainable development: Towards a new research and policy agenda Environmental Politics 16 584-603

Shawki N 2013 Understanding the transnational diffusion of social movements: an analysis of the U.S. solidarity economy network and transition US. Humanity \& Society 37 131-58

Smith A and Seyfang G 2013 Constructing grassroots innovations for sustainability Global Environmental Change 23 827-9

Soule S A 2004 Diffusion processes within and across movements in Snow D A, Soule S A and Kriesi H eds The
Blackwell companion to social movements Blackwell, Malden MA 294-310

Strang D and Soule S A 1998 Diffusion in organizations and social movements: from hybrid corn to poison pills Annual Review of Sociology 24 265-90

Tarrow S 2005 The new transnational activism Cambridge University Press, New York NY

Tarrow S 2010 Dynamics of diffusion: mechanisms, institutions, and scale shift in Givan R K, Roberts $\mathbf{K} \mathbf{M}$ and Soule $\mathbf{S} \mathbf{A}$ eds The diffusion of social movements. Actors, mechanisms, and political effects Cambridge University Press, New York NY 204-19

Truffer B and Coenen L 2012 Environmental innovation and sustainability transitions in regional studies Regional Studies 46 1-21

Van Aelst P and Walgrave S 2002 New media, new movements? The role of the internet in shaping the 'anti-globalization' movement Information, Communication Society 5 465-93

Van Laer J and Van Aelst P 2010 Internet and social movement action repertoires. Opportunities and limitations Information, Communication \& Society 13 1146-71

Walsh-Russo C 2014 Diffusion of protest Sociology Compass 8 31-42

Wilson G A 2012 Community resilience, globalization, and transitional pathways of decision-making Geoforum 43 1218-31 